\title{
study on community nursing service demand and influencing factors of elderly diabetes patients
}

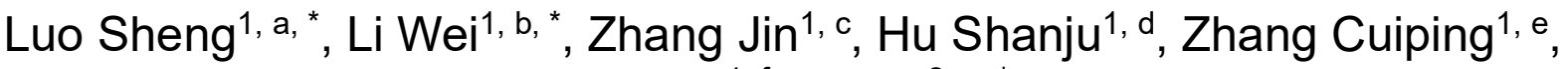 \\ Dong $\mathrm{Yi}^{1,}$, , Luo $\mathrm{Li}^{2}$, g, * \\ ${ }^{1}$ College of Public Health and Management, Weifang Medical University, Weifang, China \\ ${ }^{2}$ Weifang People's Hospital, Weifang, China \\ awfxz3418@sina.com,b1453254794@qq.com, ${ }^{\mathrm{c} 20492003 @ q q . c o m,{ }^{d} w f x z 001 @ s i n a . c o m, ~}$ \\ ezhangcuiping@wfmc.edu.cn, ${ }^{\mathrm{f}}$ dongyi@wfmc.edu.cn, ${ }^{9} 104539323 @ q q . c o m$
}

${ }^{*}$ Corresponding author

Key words: Elderly patients with diabetes; Community care; Community care needs; Influencing factors

\begin{abstract}
This study investigated the needs and influencing factors of community nursing service for elderly diabetes patients, and put forward corresponding countermeasures and suggestions to provide scientific basis for the community nursing service mode of elderly diabetes patients. A self-made questionnaire was used to investigate 520 elderly community diabetic patients in the sample area by stratified sampling. Descriptive analysis, single factor analysis and multiple stepwise linear regression analysis were used to analyze the nursing service needs and influencing factors. The study showed that the demand score of community elderly diabetes patients for health promotion was $34.18 \pm 5.084$, and the highest demand score was regular physical examination. The demand score of disease care was 20.55 \pm 5.099 , and the highest demand score was to provide fixed time for follow-up visit. The score of nursing demand for complications was $20.96 \pm 4.714$, and the highest demand was the prevention and nursing of diabetic peripheral neuropathy. The results of multi-factor analysis showed that the factors affecting the demand for health promotion care included BMI, financial source, treatment condition, VAS score, pain/discomfort, and the standard of fasting blood glucose. In the development of community care for elderly diabetes, it is necessary to clarify the diversified nursing needs of patients and the problems existing in the service process, provide multi-level and individualized community care services, so as to improve the quality of life of patients.
\end{abstract}

\section{Introduction}

Elderly diabetes mellitus is one of the chronic non-communicable diseases that seriously endanger people's health. With the development of economy and the improvement of living standard, the prevalence rate continues to rise. The onset of diabetes in the elderly is insidious, complications are many, there are a variety of senile diseases coexist and prone to hypoglycemia, if poor care will seriously affect the quality of life of patients and families [1]. Studies have shown that regular followup of patients has positive effects on the prevention of complications, blood glucose control and health behaviors. At present, for diabetes patients discharged from hospital after there has been a research in the continuity of care, but the community nursing demand for senile diabetes research is relatively broad [2]. Based on the Omaha system, health promotion model, combined with relevant domestic and foreign literature, this study designed the questionnaire of nursing needs of elderly diabetes patients in communities, and evaluated the nursing needs of patients from three aspects: health promotion, disease care and complication care. Analysis from the view of the concept of overall health of elderly patients with diabetes, the community in elderly patients with diabetes care needs, analysis of the needs of patients and the nursing problems, build elderly diabetes community service system, perfecting the community service content, make the community patients with medical personnel on the basis of demand management, make the elderly diabetes care a continuous process. Through the research on the nursing needs of the elderly diabetes patients in the community, it provides reference for the construction of the elderly diabetes care model and the development of 
nursing projects, which is of great significance to the transformation of the concept of community care service and the sustainable development of community care service for the elderly diabetes[3].

\section{Objects, contents of investigation and methods of statistical analysis}

\subsection{Subjects of investigation}

This study adopted multi-stage stratified random sampling method to collect 520 elderly diabetes patients from 12 community health service centers in shandong province. Inclusion criteria for elderly patients with diabetes were as follows: patients with diabetes diagnosed by a specialist as meeting the prevention and treatment guidelines for type 2 diabetes in China (2013 edition); Age cubed 60 years old, and live in the community for more than half a year; Clear awareness and no communication barriers, can respond correctly and answer questions; Informed consent, voluntary participation. The exclusion criteria are: those with severe cognitive impairment, confusion, uncooperation or unclear language expression; Other serious physical diseases; Serious complications of diabetes; A history of mental illness. A total of 520 questionnaires were distributed in this survey, and 500 were recovered, with a recovery rate of $96.15 \%$ and an effective recovery rate of $99.60 \%$.

\subsection{Contents of investigation}

This research tool is a self-designed questionnaire, including the basic situation of elderly diabetes patients in urban communities, Quality of life scale (EQ-5D), activity of daily living (ADL), Social Support Rating Scale (SSRS) and nursing needs of elderly diabetes patients. The general situation of the elderly, such as gender, age, marital status, educational level, occupation before retirement, medical insurance, income, chronic diseases, number of children, living style. Based on the health promotion model as the theoretical framework, the questionnaire on the nursing needs of elderly diabetes patients in urban communities divides the community nursing into health promotion, disease nursing and complication nursing, with a total of 3 dimensions and 21 items. Health promotion needs dimension has 9 items, disease care needs dimension has 6 items, complication care needs dimension has 6 items. Each item was rated on a Likert5 scale as not needing 1, not needing 2, not needing 3, needing 4, and needing 5 badly. The higher the score, the higher the nursing demand. The score of each dimension is the sum of the items of each dimension.

\subsection{Statistical analysis}

EpiData software was used to establish the database and input the data. When the data is correct, statistical analysis will be conducted after importing SPSS19.0. Description of frequency and composition ratio of counting data; Measurement data that follow normal distribution are expressed by means of mean and standard deviation. Univariate analysis was performed using $\mathrm{x}^{2}$ test, two independent samples t-test and univariate analysis of variance. Multiple factor analysis using multiple stepwise linear regression analysis, test level $\alpha=0.05$.

\section{Results}

\subsection{Basic situation of the elderly}

A total of 498 elderly patients with diabetes mellitus in urban communities were investigated in this study. Among them, 177 (35.5\%) were males and 321 (64.5\%) were females. The number of females was significantly higher than that of males. The average age was $(70.18 \pm 6.848)$ years. There were 414 married elderly diabetic patients $(83.1 \%)$ and 84 other cases (unmarried, divorced and widowed) with diabetes mellitus (16.9\%). The number of people living alone is 55 , accounting for $11.0 \%$; 53 people $(10.6 \%)$ had never attended school, 179 people $(35.9 \%)$ had primary school culture, 155 people (31.1\%) had junior high school culture, 88 people $(17.7 \%)$ had senior high school and secondary school culture, and 23 people $(4.6 \%)$ had junior college and undergraduate education. There are 365 retirees, accounting for $73.3 \%$; 19 employees, accounting for $3.8 \%$; 114 retirees, accounting for $22.9 \%$. The main sources of income for elderly diabetic patients are retirement pension, 
of which $435(87.3 \%)$ are retirement pension, $39(7.8 \%)$ are child support, $5(1.0 \%)$ are government relief, $11(2.2 \%)$ are personal savings, $2(0.4 \%)$ are income from their own work.

\subsection{Quality of life of elderly diabetes patients in urban communities}

In the poll of elderly patients with diabetes, according to EQ-5D scale for measuring quality of life situation, according to the results of action capacity problems with $22.9 \%$, self-care ability of the problems with $14.5 \%$, daily activity ability problem with $17.5 \%, 40.4 \%$ pain/discomfort, existential anxiety/depression is $22.1 \%$, according to the proportion of high and low, in turn, is pain/discomfort, action ability, anxiety/depression, daily activity ability and self-care ability. As can be seen from the table, the EQ-VAS scores of the subjects in this study were skewed distribution. For the assessment of the daily living ability of elderly diabetes patients in urban communities, 112 patients were normal, 321 patients were functional decline, $64.5 \%$, and 65 patients were functional impairment, accounting for $13.1 \%$. The differences of marital status, education level and annual income in the daily living ability of elderly diabetic patients were statistically significant.

\subsection{Social support of elderly diabetes patients}

The results showed that the mean score of social support was (35.88 \pm 4.740$)$, among which the mean score of objective support was (21.45 \pm 3.468$)$, the mean score of subjective support was $(7.37 \pm 1.480)$, and the mean score of social support was (7.07 \pm 2.078$)$. There were statistically significant differences in social support among age, education, and annual income. The social support scores of the elderly over 80 years old were the highest and those between 75 and 79 years old were the lowest. The social support scores of those who had not been to school were the highest, while those of junior college and undergraduate students were the lowest. Those with annual income of 20,000 yuan scored the highest, while those with 0 yuan scored the lowest.

\subsection{Nursing needs of elderly diabetes patients}

Among the nursing needs of elderly diabetes patients in urban communities, health promotion needs score 34.18 \pm 5.084 , disease care needs score 20.55 \pm 5.099 , complications care needs score $20.96 \pm 4.714$, and health education needs score the highest. Among the demands for health promotion, 492 people (98.8\%) needed regular physical examination. Among the demands for disease care, 381 people $(76.5 \%)$ needed to provide regular time for follow-up visits. Among the nursing needs of complications, the number of diabetic foot prevention and nursing needs was 336, accounting for $67.5 \%$.

\subsection{Influencing factors of community nursing needs}

\subsubsection{Analysis of influencing factors in health promotion dimension}

Using stepwise regression, as shown in Table1-3. The score of health promotion nursing needs was taken as the dependent variable to elderly patients with diabetes Demographic characteristics, health behavior, disease-related conditions, and quality of life were taken as independent variables to establish a multiple linear regression model. The results showed that BMI, economic source, treatment, VAS score, pain/discomfort, and fasting blood glucose were included in the model.

3.5.2 Analysis of influencing factors in the disease nursing dimension

Demography of elderly diabetics with disease care needs score as the dependent variable, and characteristics, health behaviors, disease-related conditions and quality of life were taken as independent variables, and multiple linear stepwise regression was conducted to establish multiple linear regression models. The results showed that age, education level, BMI, total number of chronic diseases, daily activity ability and fasting blood glucose level reached the standard entered the model. 3.5.3 Analysis of influencing factors in complication nursing dimension.

Multiple linear regression models were established with complication care demand score as the dependent variable and demographic characteristics, health behaviors, disease-related conditions and quality of life of elderly diabetes patients as independent variables. The results showed that age, education, BMI, fasting blood glucose, anxiety/depression entered the model 
Table 1 analysis of influencing factors of nursing needs in health promotion dimension

\begin{tabular}{ccccccc}
\hline variable & $\begin{array}{c}\text { regression } \\
\text { coefficien }\end{array}$ & $\begin{array}{c}\text { normalized } \\
\text { regression } \\
\text { coefficient }\end{array}$ & $t$ & $P$ & \multicolumn{2}{c}{ collinearity diagnosis } \\
\cline { 4 - 7 } & 36.632 & & 20.978 & 0.000 & & \\
Constant & 1.322 & 0.199 & 4.610 & 0.000 & 0.977 & 1.024 \\
BMI & -0.745 & -0.142 & -3.316 & 0.001 & 0.988 & 1.012 \\
Economic sources & -1.172 & -0.100 & -3.316 & 0.024 & 0.937 & 1.067 \\
Medical treatment & -1.995 & -0.165 & -3.719 & 0.000 & 0.922 & 1.084 \\
VAS & -1.625 & -0.158 & -3.470 & 0.001 & 0.875 & 1.143 \\
Pain/discomfort & 1.137 & 0.107 & 2.486 & 0.013 & 0.974 & 1.026 \\
Fasting blood sugar &
\end{tabular}

Table 2 Analysis on influencing factors of nursing needs in urban communities

\begin{tabular}{cccccccc}
\hline variable & $\begin{array}{c}\text { regression } \\
\text { coefficien }\end{array}$ & $\begin{array}{c}\text { normalized } \\
\text { regression } \\
\text { coefficient }\end{array}$ & $t$ & $P$ & \multicolumn{2}{c}{ collinearity diagnosis } \\
\cline { 4 - 7 } Constant & 9.816 & & 20.978 & 0.000 & & \\
age & 1.319 & 0.322 & 7.144 & 0.000 & 0.992 & 1.008 \\
BMI & 1.148 & 0.162 & 3.552 & 0.000 & 0.967 & 1.034 \\
Fasting blood sugar & 1.754 & 0.161 & 3.537 & 0.000 & 0.975 & 1.026 \\
Degree of education & -0.638 & -0.129 & -2.872 & 0.004 & 0.988 & 1.012 \\
Ability of daily activities & 1.638 & 0.113 & 2.498 & 0.013 & 0.990 & 1.010 \\
Number of chronic diseases & 0.586 & 0.106 & 2.367 & 0.018 & 0.997 & 1.003 \\
\hline
\end{tabular}

Table 3 Analysis of influencing factors in complication nursing dimension

\begin{tabular}{|c|c|c|c|c|c|c|}
\hline \multirow[b]{2}{*}{ variable } & \multirow{2}{*}{$\begin{array}{l}\text { regression } \\
\text { coefficien }\end{array}$} & \multirow{2}{*}{$\begin{array}{c}\text { normalized } \\
\text { regression } \\
\text { coefficient }\end{array}$} & \multirow[b]{2}{*}{$t$} & \multirow[b]{2}{*}{$P$} & \multicolumn{2}{|c|}{ collinearity diagnosis } \\
\hline & & & & & tolerance & $V I F$ \\
\hline Constant & 12.819 & & 10.636 & 0.000 & & \\
\hline age & 1.108 & 0.303 & 7.097 & 0.000 & 0.995 & 1.005 \\
\hline Anxiety/depression & 1.431 & 0.127 & 2.968 & 0.003 & 0.994 & 1.006 \\
\hline BMI & 0.606 & 0.097 & 2.250 & 0.025 & 0.978 & 1.022 \\
\hline Fasting blood sugar & 0.965 & 0.097 & 2.247 & 0.025 & 0.974 & 1.026 \\
\hline
\end{tabular}

\section{Discussion}

\subsection{Nursing needs of elderly diabetes patients}

Among the demands for health promotion, the proportion of people who need regular physical examination is $98.8 \%$. The second is the examination and treatment of diabetes, which shows that with the progress of the society, elderly diabetes patients have a high demand for regular physical examination, and the demand for health care awareness and knowledge is gradually increasing. This also suggests that community primary medical institutions should not only pay attention to the nursing of the disease itself, but also strengthen the publicity and education of disease related knowledge. In disease care needs, the demand is to provide the highest fixed time visit, followed by the family visit, from the perspective of the demand of the patients, we should change our ideas community nursing staff, according to the characteristics of the disease to give the guidance of the doctor, prevent the happening of the complications and development, from the "door to door service such as" to "walk out" service, the lowest for blood glucose, blood pressure, eye, foot, liver and kidney functions of monitoring, do not agree with the guangzhou research [4], which may be related to region, lifestyle, etc. In complications nursing care needs, the highest level of demand is the prevention of diabetic foot and nursing, and diabetic foot, diabetic nephropathy, diabetic eye disease needs relatively concentrated, similar to the literature results, but do not agree with the research of the city [5], may be related to objects, living habits, and complications in patients with different regional differences. In order to meet the nursing needs of patients, community medical staff should pay more attention to the cultivation of patients' self-care ability. 


\subsection{Influencing Factors on community nursing needs of elderly diabetes patients}

For the nursing demand model of health promotion dimension, BMI has a positive relationship. The higher the BMI value is, the higher the demand will be. Overweight and obese people need more diet treatment, exercise treatment and weight control. Economic sources are negatively correlated with each other. When there is a certain economic capacity, the selection is more extensive and not limited to the community. There are also similar studies. Hypoglycemic drug therapy has initiative and high demand for health promotion, which has been confirmed by investigation. VAS scores were negatively affected. Pain/discomfort is a major problem in nursing care. Elderly patients with impaired neuromuscular and skeletal functions are likely to develop peripheral neuropathy, and the demand for health promotion is reduced.

For the nursing demand model of the dimension of disease nursing, age, fasting blood glucose, daily activity capacity and the total number of chronic diseases are positively affected. With the increase of variables, the nursing demand of diseases also increases. Conversely, the need for disease care is reduced. However, the degree of education is negatively correlated. The lower the degree of education, the higher the demand for disease care, the lower the understanding of the disease, how to do, and what the nursing staff need to do, all have expectations.

The demand model of complication nursing dimension shows that age, anxiety/depression, fasting blood glucose level reaching the standard and BMI are positively correlated. These variables are all risk factors of diabetes. When complications occur, we should be more alert to the occurrence and development of complications, and patients' demands will also increase. For complications, the focus should be on the elderly, anxiety/depression, poor control of blood sugar, overweight and obese. Chinese guidelines for the prevention and treatment of type 2 diabetes suggest that elderly patients with cardiovascular diseases should fully balance the advantages and disadvantages of intensive blood glucose control and make individual choices for blood glucose control [6].

\section{Conclusion}

\subsection{Improving the model of community management of elderly diabetes}

By government policy, the health administrative department in charge of led, streets or residents' committees responsible for activity convened and provide site facilities, community health service center service platform and provide technical support, the centers for disease control in quality control and effect assessment, for the community elderly diabetes management work provide the necessary organizational guarantee[7].Community health service institutions carry out community management with the mode of general practice team, which is composed of general practitioners, community nurses, public health doctors, nutritionists and exercise rehabilitators. Ophthalmology, cardiovascular, nephropathy and psychological doctors can be added to better serve elderly patients with diabetes. Regular screening of high-risk groups, follow-up of elderly patients with diabetes, classified treatment of patients with different conditions, and a comprehensive health examination once a year.

\subsection{Improving the service content and service mode of community nursing}

According to the needs of elderly patients with diabetes status quo, reasonable increase nursing services, with reference to the Chinese type 2 diabetes prevention guide ", "national basic public health service specification (2015 edition) in the elderly patients with type 2 diabetes health management and health management basic public health services, such as standard specific service content, service process, improve the quality of service capability, community service and recognition. Starting from the three dimensions of health promotion, disease nursing and complication nursing, the model established based on the nursing needs of patients is more focused on providing services for different key groups, and community nursing services are easier to be accepted [8].

\subsection{Strengthening the training of community nurses}

At present, the community nursing education in China has just started, and the knowledge of health education about diabetes is insufficient, and the concept of compliance with medical advice is very 
strong, and the initiative is insufficient, which makes it difficult to assume the responsibilities of caregivers, managers, consultants and instructors. Community nurses should carry out professional training and further study, and professional backbone of the hospital should provide community services to improve the technical level of the community [9], increase the number of nursing staff, and improve the comprehensive quality of nursing staff.

\subsection{Strengthening the self-health management of patients}

Elderly diabetes is characterized by high prevalence, atypical symptoms, many complications and complications, low level of self-management, and easy occurrence of hypoglycemia. Selfmanagement of patients is the central link [10], including self-monitoring of blood glucose. Guide patients to carry out blood glucose monitoring at home, including monitoring techniques and monitoring methods, so as to fully grasp the control level of blood glucose and reduce the risk of hypoglycemia. Monitoring time points of blood glucose include before, after, before and after meals, at night, before and after vigorous exercise, etc. Different time points are selected according to the condition and treatment plan. Diabetes can only be controlled but not cured. It requires patients to be prepared for lifelong and long-term war against chronic diseases. Help patients establish a positive and optimistic health concept, continue to pay attention to the mental health of patients.

\section{Acknowledgement}

This research was financially supported by the Ministry of Education Social Science Research Project(Grant No.18YJCZH118); and Shandong Provincial Social Science Research Program (Grant No.18CGLJ51,No.19CGLJ29); and Shandong Provincial Natural Science Foundation(Grant No.ZR2019MG027); and Chinese Medical Association Medical Education Branch research project (Grant No.2018B-N06012,2016B-RC070); and Chinese Society of Academic Degrees and Graduate Education research project (Grant No.B-2017Y0602-092); and Humanities and social sciences Program of Shandong (Grant No.19-ZZ-GL-03)

\section{References}

[1] Erico Castro-Costa PhD,Breno S. Diniz PhD,Josélia O. A. Firmo PhD, et al. Diabetes, depressive symptoms, and mortality risk in old age: The role of inflammation. Depression and Anxiety, Vol.36, pp.941-949,2019.

[2] Lu Hongqin. Study on the "two in one" community nursing mode and effect of elderly patients with type 2 diabetes. Electronic Journal of Clinical Medical Literature, Vol.4, pp.1109$1112,2017$.

[3] Yang Fang. Effect of community nursing on quality of life and compliance of elderly diabetic patients. Electronic Journal of Practical Gynecologic Endocrinology, Vol.4, pp.176-177,2017.

[4] Teng Yun, Liu Meili, Zhang Xuemei. Application effect of hospital family integrated continuous nursing in elderly diabetic patients. Nursing Research of China, Vol.32, pp.3461-3462,2018.

[5] Chen Yingxia. The influence of community general practitioner management mode combined with traditional Chinese medicine on blood glucose and blood lipid of elderly type 2 diabetes. Modern Diagnosis and Treatment, Vol.30, pp.2883-2885,2019.

[6] Li Shuqin. Study on the practical effect of hospital community high quality nursing service in elderly patients with diabetes. Today Nurse, Vol.3, pp.34-36,2017.

[7] Li liangxiu, Yang Li, Zhu Xuemei, et al.Application of group management mode in elderly patients with type 2 diabetes in community. Journal of Nursing Science, Vol.33, pp.5-8,2018.

[8] Bo Kyung Koo,Lee-Kyung Kim,Jun-Young Lee, et al. Taking metformin and cognitive function change in older patients with diabetes. Geriatrics \&amp; Gerontology International, Vol.19, 
pp.755-761,2019.

[9] Zhu Bingying. Observation on the effect of systematic community nursing and health education for elderly diabetic patients. World Latest Medicine Information, Vol.19, pp.354-357,2019.

[10] Gong Lixiang. Evaluation of the effect of community nursing on the self-management behavior of elderly diabetic patients. Renowned Doctor, Vol.2, pp.199,2019. 